stomatologie 2013 [Suppl 1] · 110:9-9 DOI 10.1007/s00715-013-0259-4

๑) Springer-Verlag Wien 2013

\title{
Grußworte
}

\section{des Bundesministers für Gesundheit}

Im Jahr 2012 wurden von der Bundesgesundheitskommission, dem Ministerrat und dem Nationalrat zehn Rahmen-Gesundheitsziele für Österreich beschlossen. Sie sollen ganz konkret dazu beitragen, dass in den nächsten 20 Jahren die in Gesundheit verbrachten Lebensjahre im Durchschnitt um 2 Jahre steigen.

$\mathrm{Zu}$ einem langen Leben bei guter $\mathrm{Ge}$ sundheit und hoher Lebensqualität gehören auch gesunde und schöne Zähne bis ins hohe Alter. Da Zahn- und Zahnfleischerkrankungen nachteilige Auswirkungen auf die Gesamtgesundheit haben können, ist mir die Integration von Mundgesundheit in Präventions- und Gesundheitsförderungsprogramme ein wichtiges Anliegen. Dank der Fortschritte in der Zahnmedizin und durch verstärkte Bemühungen in der Prophylaxe hat sich die Zahngesundheit der Österreicher in den letzten Jahrzehnten deutlich verbessert.

Immer neue Entwicklungen und technische Fortschritte, Präventionsorientierung und Alterszahnheilkunde stellen die Zahnheilkunde im 21. Jahrhundert jedoch vor große Herausforderungen. Um den Patienten die bestmögliche Behandlung anbieten zu können, kommt in einem sich rasant weiterentwickelnden Fachgebiet wie der Zahnheilkunde der qualitätsgesicherten Fortbildung ein hoher Stellenwert zu.
Die Österreichische Gesellschaft für Zahn- Mund- und Kieferheilkunde, die im Jahr 1861 mit dem Ziel der wissenschaftlichen Fortbildung ihrer Mitglieder von dem Wiener Zahnarzt Moriz Heider gegründet wurde, hat in diesem Sinne eine verantwortungsvolle Aufgabe übernommen. Denn der Bedarf nach wissenschaftlich gesicherten Informationen im Rahmen einer unabhängigen zahnärztlichen Fortbildung ist heute - 150 Jahre nach der Gründung der ÖGZMK größer denn je. Als Gesundheitsminister wünsche ich diesem traditionsreichen Verein, der sich abseits aller politischen Interessen der wissenschaftlichen Weiterentwicklung verpflichtet fühlt, weiterhin ein erfolgreiches Wirken zum Wohle der Patienten.

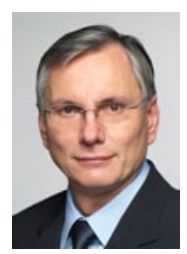

Alois Stöger

Bundesminister für Gesundheit 